\title{
A Gene Differentially Expressed in the Kidney of the Spontaneously Hypertensive Rat Cosegregates with Increased Blood Pressure
}

\author{
Nilesh J. Samani, ${ }^{\star}$ David Lodwick, ${ }^{\star}$ Madeleine Vincent, ${ }^{\star}$ Christopher Dubay, ${ }^{3}$ Michael A. Kaiser, ${ }^{\star}$ Martin P. Kelly, \\ Ming Lo, ${ }^{\ddagger}$ Janet Harris, ${ }^{\star}$ Jean Sassard, ${ }^{\ddagger}$ Mark Lathrop, \\ ${ }^{*}$ Department of Medicine, University of Leicester, Leicester LE2 $7 L X$, United Kingdom, ${ }^{\ddagger}$ URA Centre National de la Recherche \\ Scientifique 1483, Departement de Physiologie et de la Pharmacologie Clinique, Faculte de Pharmacie, 69373, Lyon, France; and \\ 'Institut National de la Sante et de la Recherche Médicale U. 358, Centre d'Étude du Polymorphism Humain, 75010, Paris, France
}

\begin{abstract}
The role of the kidney in initiating hypertension has been much debated. Here we demonstrate that a recently identified gene of yet unknown function, termed $S_{A}$, which is differentially expressed in the kidney of the spontaneously hypertensive rat, cosegregates with an increase in blood pressure in $F_{2}$ rats derived from a cross of the spontaneously hypertensive rat with normotensive Wistar-Kyoto rats, accounting for 28 and $21 \%$ of the genetic variability in systolic and diastolic blood pressures, respectively. Further, the genotype at this locus appears to determine the level of expression of the gene in the kidney. The findings provide strong evidence for a primary genetic involvement of the kidney in hypertension. (J. Clin. Invest. 1993. 92:1099-1103.) Key Words: hypertension • genetics • kidney • spontaneously hypertensive rat • gene expression
\end{abstract}

\section{Introduction}

The mapping of genetic loci underlying simple mendelian traits has now become readily achievable. For polygenic traits, especially those with additional environmental determinants such as hypertension (1) and atheroma (2), major difficulties still remain. However, the feasibility of mapping genetic loci directly involved in blood pressure regulation has recently been demonstrated in a number of rodent models of genetic hypertension (3-9) where a limited number of genes control the hypertensive phenotype (10). Nevertheless, in most cases the mechanisms by which the linked loci influence blood pressure remain to be determined, and no intermediate phenotypes have as yet been identified. Recently, a gene, designated $S_{A}$, demonstrating increased expression in the kidney of the spontaneously hypertensive rat (SHR $)^{1}$ compared with its normotensive control, the Wistar-Kyoto (WKY) rat, has been identified by differential hybridization (11). The kidney plays a central

Address correspondence to Dr. N. J. Samani, Department of Medicine, University of Leicester, Clinical Sciences Building, Leicester Royal Infirmary, P. O. Box 65, Leicester LE2 7LX, United Kingdom.

Received for publication 12 March 1993 and in revised form 30 April 1993.

1. Abbreviations used in this paper: LOD, likelihood of the odds; MT1PA, metallothionein-1 pseudogene a; SHR, spontaneously hypertensive rat; WKY, Wistar-Kyoto rat.

J. Clin. Invest.

(C) The American Society for Clinical Investigation, Inc.

0021-9738/93/08/1099/05 \$2.00

Volume 92, August 1993, 1099-1103 role in blood pressure regulation (12-14), and there is evidence from transplantation studies of its involvement in both genetic hypertension in rats (15-17) and in human essential hypertension $(13,14)$. We therefore undertook a study to investigate a possible role for the $\mathrm{S}_{\mathrm{A}}$ gene in the etiology of hypertension in the SHR. In addition to carrying out cosegregation analysis of alleles at the locus with blood pressure in $F_{2}$ rats derived from a cross of SHR and WKY rats, we also determined the influence of the genotype at the locus on $S_{A}$ mRNA levels in the kidney.

\section{Methods}

Generation of $F_{2}$ rats. 3 SHR male rats and 3 SHR female rats were reciprocally mated with the same number of WKY rats (all animals obtained from the breeding stock of Charles River Laboratories, Margate, UK) to obtain $F_{1}$ hybrids. $F_{1}$ animals from each half of the cross were randomly mated to generate 233 male $F_{2}$ rats. All animals were housed under controlled conditions (temperature $21 \pm 1^{\circ} \mathrm{C}$, humidity $60 \pm 10 \%, 12$-h day/night cycle), fed standard rat chow containing $0.25 \% \mathrm{Na}$ and $0.66 \% \mathrm{~K}$ (Rat \& Mouse No. 3 Breeding Diet; Special Diet Services Ltd., Witham, Essex, UK), and given free access to tap water.

Blood pressure measurements. Indirect systolic blood pressure in tail artery was measured at 12,16 , and $20 \mathrm{wk}$ of age after prewarming of the animals to $32^{\circ} \mathrm{C}$. A $7 / 16$-in tubular cuff (Linton Instrumentation, Diss, UK) was used to occlude the artery and a pneumatic sensor and pulse transducer (Physiograph; Narco Bio Systems Inc., Houston, TX) connected to an oscilloscope to detect the pulsations on release of the cuff. Direct blood pressure measurements were obtained at $25 \pm 1 \mathrm{wk}$ of age using a modification of our computerized technique (18). Under anesthesia, a catheter was inserted via the femoral artery into the lower abdominal aorta, and the rat was placed into an individual recording cage and allowed to recover for $24 \mathrm{~h}$. The arterial catheter was connected to a blood pressure transducer (Statham P23ID; Gould Inc., Cleveland, $\mathrm{OH}$ ) via a rotating swivel that allowed the animals to be unrestrained. Calibration of the instrument was verified before each measurement. Blood pressure measurements were recorded beat by beat for 2 consecutive $h$ between 1000 and 1700 . The data were processed off-line following the method of Gustin et al. (19).

Genotyping. DNA typing was carried out for a StuI polymorphism at the $S_{A}$ locus (20). DNA was prepared from tail fragments as described (21) and Southern blot analysis carried out using standard protocols (22). Probe was a 1.6-kb $\mathrm{S}_{\mathrm{A}} \mathrm{cDNA}$ subcloned from a fragment isolated by reverse transcription and PCR amplification (23) of 6-wkold SHR kidney total RNA using primers based on the published sequence ( 11) (5':TGGCTTTCTCTCCCATTAAG (nucleotides 7-26); 3':TGTTGTCCATTCCTTTCTCC (nucleotides 1904-1923). Authenticity of the amplified fragment was confirmed by restriction mapping and sequencing of the ends of the clone (22).

$R N A$ analysis. Total kidney RNA was prepared by $\mathrm{LiCl} /$ urea precipitation (24) and $60-\mu \mathrm{g}$ aliquots analyzed using standard Northern blot techniques (22). The probes used were the $S_{A}$ cDNA fragment 
described above and an HGPRT cDNA (25). Genotypes of the $F_{2}$ rats used in this analysis were ascertained as described above.

Statistical analysis. Statistical analysis was carried out using MINITAB (Release 7) (Minitab Inc., University Park, PA) and the LINKAGE program (26).

\section{Results}

$S_{A}$ polymorphism cosegregates with blood pressure. 233 male $F_{2}$ rats were generated from the SHR $\times$ WKY cross. All animals had indirect tail cuff blood pressures measured at 12,16 , and 20 wk of age. In addition, 193 randomly chosen animals had direct blood pressures measured at $25 \pm 1$ wk of age. 16 male SHR and WKY rats were also studied in a similar fashion.

Fig. 1 illustrates the various $S_{A}$ genotypes observed in the $F_{2}$ rats, and Table I shows the direct blood pressures at 25 wk of age of $F_{2}$ rats, according to the $S_{A}$ genotypes. As can be seen, the $S_{A}$ locus had a highly significant effect on both systolic and diastolic blood pressures. The effect of the SHR allele was codominant and the locus accounted for 11 and $10 \%$ respectively of the total variance of systolic and diastolic blood pressures in the $F_{2}$ population.

The total variances of systolic and diastolic blood pressures in $F_{2}$ rats are the sums of their respective genetic and environmental variances. The degrees of genetic determination (DGD $=$ ratio of genetic to total variance) of systolic and diastolic blood pressures in the $F_{2}$ rats were calculated as described by Rapp (10) using the variances of the respective parental blood pressures as estimates of the environmental variances. The values calculated for DGD $( \pm \mathrm{SE})$ were $39 \pm 15 \%$ for systolic blood pressure and $46 \pm 12 \%$ for diastolic blood pressure, therefore suggesting that the locus accounts for up to $28 \%$ of the genetic variance of systolic blood pressure and $21 \%$ of the genetic variance of diastolic blood pressure in the $F_{2}$ population.

Longitudinal effects on blood pressure. The blood pressure of the $\mathrm{F}_{2}$ rats rose progressively from 12 to $20 \mathrm{wk}$ of age ( $12 \mathrm{wk}$ : $144.00 \pm 0.79$; 16 wk: $151.85 \pm 0.86 ; 20$ wk: $158.98 \pm 0.99$ ) with highly significant correlations between blood pressures at the different ages (data not shown). In addition, blood pressures at 25 wk correlated significantly with the indirect blood pressures at all ages with, as expected, increasing correlation from 12 to $20 \mathrm{wk}(r=0.378,0.526$, and 0.574 for mean arterial pressure at 25 wk and 12-, 16-, and 20-wk BPs, respectively; $P<0.0001$ in all cases). The effect of the $S_{A}$ locus on the longitudinally measured indirect blood pressures is shown in Fig. 2. The effect appears to be age dependent, only becoming significant at 20 wk of age. While this finding needs to be interpreted with some caution because of the indirect method of measuring blood

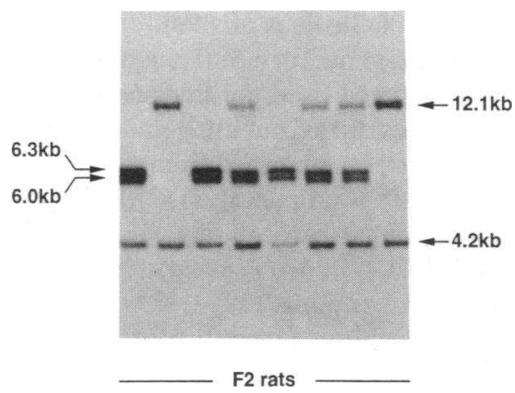

Figure 1. Southern blot showing the $\mathrm{F}_{2}$ genotypes at the $S_{A}$ locus identified by StuI. Results of the typing of eight individual rats are shown. In addition to the constant band ( 4.2 $\mathrm{kb})$, a single band at $12.1 \mathrm{~kb}$ (e.g., tracks 2 and 8 ) indicates homozygosity for the SHR

allele (S genotype) while the presence of two bands at $6.0 / 6.3 \mathrm{~kb}$ (e.g., tracks 1 and 3 ) homozygosity for the WKY allele (W genotype).
Table I. Linkage of $S_{A}$ Gene to Blood Pressure Phenotypes

\begin{tabular}{lrcc}
\hline Genotype & No & SBP & DBP \\
\hline W & 49 & $179.0 \pm 1.8$ & $115.3 \pm 1.3$ \\
H & 100 & $184.1 \pm 1.4$ & $120.1 \pm 1.1$ \\
S & 44 & $192.6 \pm 2.0$ & $125.0 \pm 1.4$ \\
F value (ANOVA) & 11.7 & 10.3 \\
$\begin{array}{l}P \text { value (ANOVA) } \\
\text { LOD score statistic* (at }\end{array}$ & $<0.0001$ & $<0.0001$ \\
0\% recombination) & & 3.8 \\
\end{tabular}

Linkage of $S_{A}$ gene to blood pressure phenotypes in $F_{2}$ rats derived from an SHR $\times$ WKY cross. W, homozygous for WKY allele; $H$, heterozygous; S, homozygous for SHR allele; SBP, systolic blood pressure; DBP, diastolic blood pressure.

* Base 10 logarithm of the likelihood ratio statistic for the test of linkage.

pressure, it emphasizes the importance of considering age as a factor when investigating the effect of a gene on blood pressure.

Chromosomal location. The chromosomal location of the rat $S_{A}$ gene was established by linkage analysis with other polymorphic markers typed in the $F_{2}$ rats whose chromosomal locations had previously been established (27). Significant linkage was found between $S_{A}$ and a microsatellite marker $R 231$ of the rat metallothionein-1 pseudogene a (MT1PA) located on chromosome 1 (27) with a maximum likelihood of the odds (LOD) score of 44.8 at $7.3 \%$ recombination. This marker was also associated with increased blood pressure, but the linkage was much weaker than that found with the $S_{A}$ locus (Table II). Two other informative markers located elsewhere on rat chromosome 1 (27) showed no linkage with either $S_{A}$ or MT1PA or with blood pressure (Table II).

$S_{A}$ genotype and kidney expression. The $\mathrm{S}_{\mathrm{A}}$ gene shows very limited tissue distribution of expression, with most marked expression in the kidney and some expression in the liver and brain (11, and unpublished data of our own). In the SHR kidney it also shows age-related changes in expression, with low levels before $4 \mathrm{wk}$ of age and then a dramatic ( $>10$-fold) rise that is maintained up to at least $16 \mathrm{wk}$ of age (11). This pattern parallels closely the rise in blood pressure in the SHR (28). In the WKY kidney, expression remains constant with age and after $4 \mathrm{wk}$ is $<10 \%$ of that in the SHR kidney. To determine whether the level of expression in the kidney was directly controlled by the $S_{A}$ locus, we examined $S_{A}$ mRNA levels in kidneys of $15 F_{2}$ rats with the various $S_{A}$ genotypes. As shown in Fig. 3 the $S_{A}$ locus seems itself to be the main determinant of the level of $S_{A}$ gene expression in the kidney with the SHR allele again, as in the case of blood pressure, acting in a codominant fashion.

Heterogeneity of $S_{A}$ transcripts. Some heterogeneity was seen between SHR and WKY in the size of the kidney $S_{A}$ gene transcripts detected by Northern blotting (Fig. 3). This has also been previously reported (11). To investigate the possibility that this heterogeneity was due to differences in the coding region, PCR amplification of SHR and WKY kidney total RNA was carried out using primers flanking the coding region. In both cases a single fragment of the size predicted from the published sequence (11) was detected (data not shown). This indicates, first, that at least one of the mRNA species detected 


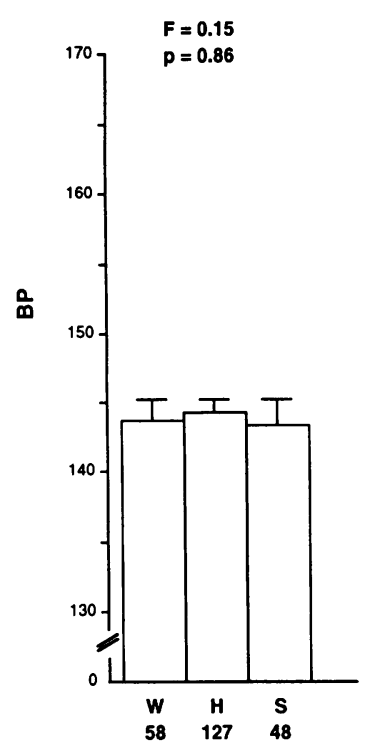

12 weeks

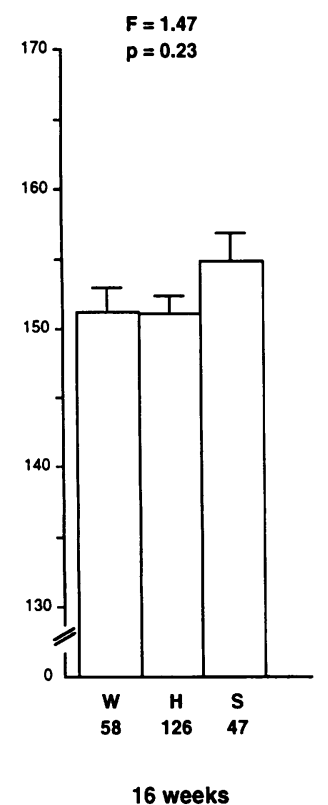

16 weeks

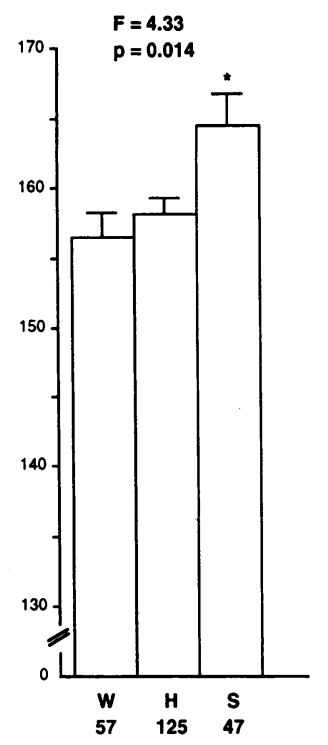

20 weeks

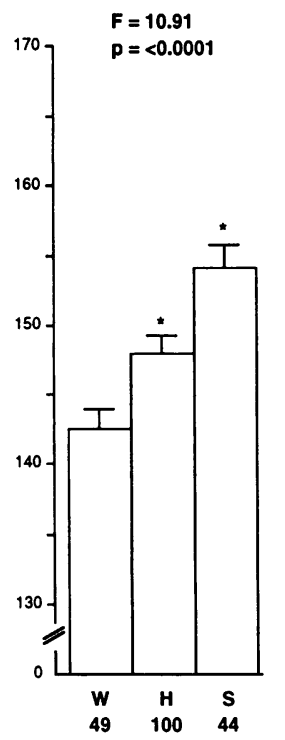

25 weeks
Figure 2. Age-dependent effects of the $S_{A}$ gene on blood pressure in $F_{2}$ rats from SHR $\times$ WKY cross. See Methods for experimental details. W, homozygous for WKY allele; $\mathrm{H}$, heterozygous; $\mathrm{S}$, homozygous for SHR allele. The numbers below each label indicate the number of animals in that group. In addition to the indirect blood pressures at 12,16 , and $20 \mathrm{wk}$, the direct mean arterial blood pressures at 25 wk are also shown. $\mathrm{F}$ and $P$ values are from ANOVA. *Significant difference $(P<0.01)$ from other groups by pairwise comparisons using Scheffe's test. by Northern blotting in the WKY is an $\mathrm{S}_{\mathrm{A}}$ gene transcript, and second, that the heterogeneity is not due to a difference between the coding regions of the SHR and WKY transcripts. The heterogeneity may therefore be due to differences in the noncoding regions, or one of the transcripts may be the product of a separate but related gene. The absence of a similar transcript in the SHR (Fig. 3) and Southern blot analysis of SHR and WKY DNA (data not shown) however argues against the presence of a related gene.

\section{Discussion}

Hypertension is a common disorder (prevalence $\sim 15 \%$ ) of largely unknown cause. It is the main risk factor for stroke and significantly influences the risk of ischemic heart disease, heart failure, kidney failure, and peripheral vascular disease (1). Family studies have demonstrated that hypertension is a multifactorial trait with a strong genetic component $(1,10,29)$. The identification of the major genetic determinants of hypertension would enable both preventive and therapeutic maneuvers to be targeted more precisely and thus improve the management of the condition.

Studies of the genetics of human hypertension are complicated by the late onset of hypertension, the small sizes of human pedigrees, and the impact of environmental effects, in addition to the likely heterogeneity of the disease in human populations $(1,10,29)$. Nevertheless, the locus that causes a rare form of inherited hypertension (glucocorticoid-remedial hypertension) has recently been identified (30) and affected sibship analysis has implicated the angiotensinogen locus in essential hypertension (31).

Inbred rodent models of genetic hypertension, with their higher heritability of blood pressure and uniform genetic causes within each strain and cross, provide an attractive alternative to identifying genes that regulate blood pressure (10). Using molecular approaches, several loci that influence blood pressure have recently been identified in a number of such strains (3-9). In most cases however, the mechanisms (socalled intermediary phenotypes) through which blood pressure is affected remain unclear.

Table II. Linkage of Other Chromosome 1 Markers to Blood Pressure Phenotypes

\begin{tabular}{|c|c|c|c|c|c|c|}
\hline & \multicolumn{6}{|c|}{ Locus } \\
\hline & \multicolumn{2}{|c|}{ MTIPA } & \multicolumn{2}{|c|}{$\mathrm{KAL}$} & \multicolumn{2}{|c|}{ PKC } \\
\hline & SBP & DBP & SBP & DBP & SBP & DBP \\
\hline W & $181.7 \pm 2.2$ & $117.8 \pm 1.6$ & $183.0 \pm 2.1$ & $119.2 \pm 1.6$ & $182.7 \pm 2.1$ & $118.2 \pm 1.5$ \\
\hline $\mathbf{H}$ & $182.5 \pm 1.4$ & $119.1 \pm 1.2$ & $184.5 \pm 1.5$ & $120.2 \pm 1.1$ & $184.2 \pm 1.5$ & $119.6 \pm 1.2$ \\
\hline$S$ & $191.3 \pm 1.9$ & $124.2 \pm 1.4$ & $187.6 \pm 2.4$ & $122.2 \pm 1.8$ & $186.1 \pm 2.3$ & $122.0 \pm 1.7$ \\
\hline$F$ value & 7.2 & 4.6 & 1.0 & 0.8 & 0.5 & 1.2 \\
\hline$P$ & 0.001 & 0.011 & NS & NS & NS & NS \\
\hline LOD score & 3.0 & 1.8 & & & & \\
\hline
\end{tabular}

Linkage of other rat chromosome 1 markers to blood pressure phenotypes in $F_{2}$ rats. 15 loci located on rat chromosome 1 (27) were screened for microsatellite polymorphism between SHR and WKY DNA as previously described (27). Three were found to be polymorphic (MTIPA, renal kallikrein [KAL], protein kinase $C$ type $1[P K C]) . F_{2}$ rats were then typed for these loci. The table shows the blood pressures of the $F_{2}$ rats according to the genotypes at each locus. Abbreviations as per Table $\mathrm{I}$. 


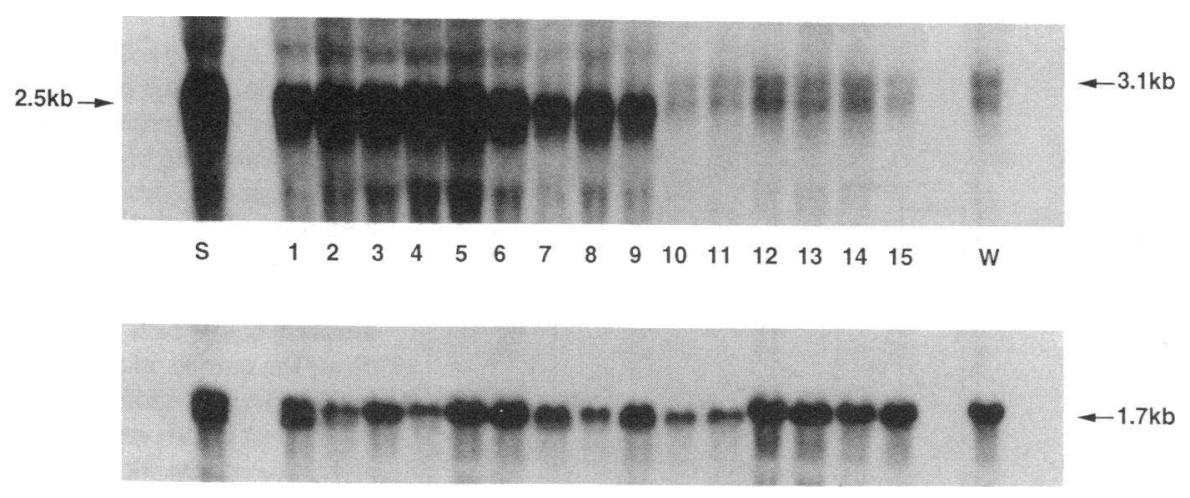

Figure 3. Effect of $\mathrm{S}_{\mathrm{A}}$ genotype on kidney $S_{A}$ mRNA levels at 6 wk of age. A Northern blot of kidney RNA was prepared from 6-wk-old SHR (S), WKY (W), and $15 \mathrm{~F}_{2}$ rats, probed for $\mathrm{S}_{\mathrm{A}}$ mRNA (top) and then reprobed for HGPRT mRNA (bottom). $\mathrm{F}_{2}$ rats 1-6 had $S$ genotype, 7-9 had $\mathrm{H}$ genotype, and 10-15 had W genotype. Despite some variation in the levels of the control HGPRT mRNA, the data demonstrate the marked effect of the $S_{A}$ genotype on kidney $S_{A}$ mRNA levels (2.5-kb band) at 6 wk of age. An additional 3.1-kb species was also detected in kidneys from animals with the W genotype (see text for discussion).
In this study, we demonstrate that a polymorphism in a gene that shows increased expression in the kidney of the SHR strongly cosegregates with raised blood pressure. While we cannot definitively exclude the possibility that a closely linked locus is responsible for the effect, the data strongly point to the $S_{A}$ gene itself. Additional support for a direct involvement of $S_{A}$ is provided by the observations ( 11 ) that its expression is modulated by antihypertensive treatment (with captopril) in the SHR and that, at least in some strains, it is also affected by dietary salt intake, a factor well known to influence blood pressure. Cosegregation of the locus with blood pressure has also recently been reported in other smaller crosses involving the SHR $(20,32,33)$. Located on chromosome 1 , the $S_{A}$ gene is not linked to any of the other loci that have been reported to cosegregate with blood pressure in previous crosses involving the SHR or the closely related stroke-prone SHR (4-9). In previous studies of this type, linkage of specific loci to blood pressure phenotypes has been found to be cross-dependent (3, 4,9 ), and whether the $S_{A}$ locus plays a role in the elevated blood pressure of other strains of genetically hypertensive rats (e.g., Dahl, Milan) remains to be determined.

We further show that the $S_{A}$ genotype also influences the level of $S_{A}$ mRNA in the kidney. It is important to note that the effect of $S_{A}$ on its kidney expression was manifested by $6 \mathrm{wk}$ of age, while the effect on blood pressure was not significant till beyond 16 wk of age. This implies that the difference seen in kidney $S_{A}$ expression is not secondary to a change in systemic blood pressure. We have therefore identified a single gene locus that affects blood pressure and also appears to directly influence its expression in a key cardiovascular tissue. Lower levels of expression of $S_{A}$ also occur in the liver and brain (11, 32, and our unpublished data). The level of $S_{A}$ mRNA is also higher in the SHR liver but lower in SHR brain compared with WKY $\left(11,32\right.$, and our unpublished data). While an effect of the $S_{A}$ locus on blood pressure through expression in these sites cannot at present be ruled out, especially as antihypertensive treatnınt (with captopril) has been reported to have a more pronounced effect on liver than kidney $S_{A}$ mRNA levels (11), our findings together with the limited tissue distribution of expression of the gene and its temporal pattern in the SHR (11) suggest that the effect of the $S_{A}$ gene locus on blood pressure is mediated via an effect on $S_{A}$ gene expression in the kidney.

The findings particularly bear upon previous observations made in experiments involving transplantation of SHR and WKY kidneys $(16,17)$. These studies have shown that the tendency to hypertension follows the SHR kidney and that this is not due to secondary changes in the SHR kidney as a conse- quence of increased blood pressure. Our findings offer a potential genetic mechanism for these physiological observations. The relevance of our findings to human hypertension remains to be investigated although it is notable that the tendency to hypertension in human kidney recipients has also been shown to be related to the blood pressure status of the donor $(13,14)$.

The function of the $S_{A}$ gene product is unknown. The nucleotide sequence of the SHR $S_{A}$ cDNA suggests a putative protein comprising 546 amino acids (11). The primary sequence has little homology to any other known protein sequence. The $S_{A}$ protein therefore likely represents a hitherto unidentified molecule involved in blood pressure homeostasis. The disparate temporal effects of the $S_{A}$ genotype on kidney expression and blood pressure suggest that if the effect of $S_{A}$ gene on blood pressure is mediated through its renal expression, then the mechanism involved is slow and/or indirect. In this context it may be relevant that an increased renal vascular resistance in young $F_{2}$ rats derived from an SHR $\times$ WKY cross has been shown also to cosegregate with hypertension (34). At present, however, the localization of the $S_{A}$ gene product in the kidney is not known. Determining this and elucidating its mechanism of action may not only provide clues to the etiology of human essential hypertension but also offer novel therapeutic options.

\section{Acknowledgments}

We thank V. Orea, P. Privat, L. Dubay, Dr. D. Forbes, and the staff of the Biomedical Services Unit, Leicester University, for their technical assistance.

We gratefully acknowledge support from the Wellcome Trust, British Heart Foundation, and the Centre National de la Recherche Scientifique. N. J. Samani, D. Lodwick, M. Vincent, J. Sassard, M. Lathrop, and J. D. Swales are members of the EURHYPGEN Concerted Action of the EEC.

\section{References}

1. Ward, R. 1990. Familial aggregation and genetic epidemiology of blood pressure. In Hypertension: Pathophysiology, Diagnosis and Management. J. H. Laragh and B. M. Brenner, editors. Raven Press, New York. 81-100.

2. Ross, R. 1986. The pathogenesis of atherosclerosis: an update. N. Engl. J. Med. 314:488-500.

3. Rapp, J. P., S. M. Wang, and H. Dene. 1989. A genetic polymorphism in the renin gene of Dahl rats cosegregates with blood pressure. Science (Wash DC). 243:542-544.

4. Kurtz, T. W., L. Simonet, P. M. Kabra, S. Wolfe, L. Chan, and B. L. Hjelle. 1990. Cosegregation of the renin allele of the spontaneously hypertensive rat with an increase in blood pressure. J. Clin. Invest. 85:1328-1332.

5. Pravenec, M., L. Simonet, V. Kren, J. Kunes, G. Levan, J. Szpirer, C. Szpirer, and T. Kurtz. 1991. The rat renin gene: assignment to chromosome 13 and linkage to the regulation of blood pressure. Genomics. 9:466-472. 
6. Pravenec, M., V. Kren, J. Kunes, A. G. Scicli, O. A. Carretero, L. Simonet, and T. W. Kurtz. 1991. Cosegregation of blood pressure with a kallikrein gene family polymorphism. Hypertension (Dallas). 17:242-246.

7. Hilbert, P., K. Lindpainter, J. S. Beckmann, T. Serikawa, F. Soubrier, C. Dubay, P. Cartwright, B. De Gouyon, C. Julier, S. Takahasi, et al. 1991. Chromosomal mapping of two genetic loci associated with blood-pressure regulation in hereditary hypertensive rats. Nature (Lond.). 353:521-529.

8. Jacob, H. J., K. Lindpainter, S. E. Lincoln, K. Kusumi, R. K. Bunker, Y. P. Mao, D. Ganten, V. J. Dzau, and E. S. Lander. 1991. Genetic mapping of a gene causing hypertension in the stroke-prone spontaneously hypertensive rat. Cell. 67:213-224.

9. Deng, Y., and J. P. Rapp. 1992. Cosegregation of blood pressure with angiotensin converting enzyme and natriuretic peptide receptor genes using Dahl salt-sensitive rats. Nature Genetics. 1:267-272.

10. Rapp, J. P. 1983. Genetics of experimental and human hypertension. In Hypertension, Physiopathology and Treatment. J. Genest, O. Kuchel, P. Hamet, and M. Cantin, editors. McGraw-Hill, Inc., New York. 582-598.

11. Iwai, N., and T. Inagami. 1991. Isolation of preferentially expressed genes in the kidneys of hypertensive rats. Hypertension (Dallas). 17:161-169.

12. Hall, J. E., H. L. Mizelle, D. A. Hildebrandt, and M. W. Brands. 1990. Abnormal pressure natriuresis. A cause or a consequence of hypertension? $\mathrm{Hy}$ pertension (Dallas). 15:547-559.

13. deWardener, H. E. 1990. The primary role of the kidney and salt intake in the aetiology of hypertension: part I. Clin. Sci. (Lond.). 79:193-200.

14. deWardener, H. E. 1990. The primary role of the kidney and salt intake in the aetiology of hypertension: part II. Clin. Sci. (Lond.). 79:289-297.

15. Bianchi, G., U. Fox, G. F. DiFranseco, A. M. Giovantti, and D. Pagetti. 1972. Blood pressure changes produced by kidney cross-transplantation between spontaneously hypertensive rats and normotensive rats. Clin. Sci. Mol. Med. 47:435-448.

16. Rettig, R., H. Stauss, C. Folberth, D. Ganten, B. Waldherr, and T. Unger. 1989. Hypertension transmitted by kidneys from stroke-prone spontaneously hypertensive rats. Am. J. Physiol. 257:F197-F203.

17. Rettig, R., C. Folberth, H. Strauss, D. Kopf, R. Waldherr, and T. Unger. 1990. Role of the kidney in primary hypertension: a renal transplantation study. Am. J. Physiol. 258:F606-F611.

18. Su, D. F., C. Cerutti, C. Barres, M. Vincent, and J. Sassard. 1986. Blood pressure and baroreflex sensitivity in conscious hypertensive rat of Lyon strain. Am. J. Physiol. 251:H1111-H1117.

19. Gustin, M. P., C. Cerutti, and C. Z. Paultre. 1990. Heterogeneous computer network for real-time hemodynamic signal processing. Comput. Biol. Med. 20:205-215.

20. Iwai, N., and T. Inagami. 1992. Identification of a candidate gene responsi- ble for the high blood pressure of spontaneously hypertensive rats. J. Hypertens. 10:1155-1157.

21. Samani, N. J., W. J. Brammar, and J. D. Swales. 1989. A major structural abnormality in the renin gene of the spontaneously hypertensive rat. J. Hypertens. 7:249-254.

22. Sambrook, J., E. F. Fritsch, and T. Maniatis. 1989. Molecular Cloning: A Laboratory Manual. Cold Spring Harbor Laboratory, Cold Spring Harbor, NY.

23. Kawasaki, E. S. 1990. Amplification of RNA. In PCR protocols: A Guide to Methods and Applications. M. A. Innis, D. H. Gelfand, J. J. Sninsky, and T. J. White, editors. Academic Press, London. 21-27.

24. Samani, N. J., J. D. Swales, and W. J. Brammar. 1988. Expression of the renin gene in extra-renal tissues of the rat. Biochem. J. 253:907-910.

25. Brennand, J., D. S. Konecki, and C. T. J. Caskey. 1983. Expression of human and Chinese hamster hypoxanthine-guanine phosphoribosyltransferase cDNA recombinants in cultured Lesch-Nyhan and Chinese hamster fibroblasts. J. Biol. Chem. 258:9593.

26. Lathrop, G. M., and J. M. Lalouel. 1984. Easy calculations of lod scores and genetic risks on small computers. Am. J. Hum. Genet. 36:460-465.

27. Serikawa, T., T. Kuramoto, P. Hilbert, M. Mori, J. Yamada, C. J. Dubay, K. Lindpainter, D. Ganten, J. L. Guenet, G. M. Lathrop, and J. S. Beckman. 1992. Rat gene mapping using PCR-analyzed microsatellites. Genetics. 131:701721.

28. Yamori, Y. 1983. Physiopathology of the various strains of spontaneously hypertensive rats. In Hypertension. J. Genest, O. Kuchel, P. Hamet, and P. Cantin, editors. McGraw-Hill, Inc., New York. 556-581.

29. Mongeau, J. G. 1989. Heredity and blood pressure. Semin. Nephrol. 9:208-216.

30. Lifton, R. P., R. G. Dluhy, M. Powers, G. M. Rich, S. Cook, S. Ulick, and J. M. Lalouel. 1992. A chimaeric 11 beta-hydroxylase/aldosterone synthase gene causes glucocorticoid-remediable aldosteronism and human hypertension. $\mathrm{Na}$ ture (Lond.). 355:262-265.

31. Jeunemaitre, X., F. Soubrier, Y. V. Koteletsev, R. P. Lifton, C. S. Williams, A. Charru, S. C. Hunt, P. N. Hopkins, R. R. Williams, J. M. Lalouel, and P. Corvol. 1992. Molecular basis of human hypertension: role of angiotensinogen. Cell. 71:169-180

32. Iwai, N., T. W. Kurtz, and T. Inagami. 1992. Further evidence of the SA gene as a candidate gene contributing to the hypertension in spontaneously hypertensive rat. Biochem. Biophys. Res. Commun. 188:64-69.

33. Lindpainter, K., P. Hilbert, D. Ganten, B. Nadal-Ginard, T. Inagami, and $N$. Iwai. 1993. Molecular genetics of the $S_{A}$ gene: co-segregation with hypertension and mapping to rat chromosome 1. J. Hypertens. 11:83-88.

34. Harrap, S. B., and A. E. Doyle. 1988. Genetic co-segregation of renal haemodynamics and blood pressure in the spontaneously hypertensive rat. Clin. Sci. (Lond.). 74:63-69. 